\title{
An Application of the Direct Method of Liapunov in Stability Analysis of Semilinear Systems
}

\author{
Igor Dobovšek \\ Faculty of mathematics and physics \\ University of Ljubljana \\ Jadranska 19, 1000 Ljubljana, Slovenia
}

\begin{abstract}
An application of the direct method of Liapunov for establishing stability criteria of smooth solutions for a certain class of initial boundary value problems described by a system of semi-linear hyperbolic partial differential equations is discussed. The concept of Liapunov-like functional is utilized to investigate some qualitative and quantitative stability properties of classical solutions for a system of semi-linear partial differential equations. The advantage of the method is that it does not require the explicit knowledge of solutions. This feature may have a significant impact on practical applications.
\end{abstract}

Keywords-quasi-linear PDEs; liapunov's functional; sign definite integral form; asymptotic stability

\section{INTRODUCTION}

Stability analysis by the Liapunov's direct method has been applied extensively to ordinary differential equations and its theoretical background pertains to stability investigation of ordinary differential equations. The extension of fundamental ideas behind it to systems of partial differential equations requires a generalization of basic principles to appropriate function spaces where a motion over a finite or infinite time interval is considered as an evolution of diffeomorphisms governed by a system of hyperbolic semi-linear partial differential equations. The essential feature of the second or the direct method of Liapunov is that the qualitative aspects of evolution of solutions that define the stability of the system are determined by means of an auxiliary function whose properties are established directly in accord with the theoretical framework of the theory itself without recourse to the solution of the system. To determine stability which in some cases implies existence, we do not need to know the actual solution of the problem. The most difficult part of the procedure is the construction of the Liapunov's functional. There is no general or unique way to find or construct the appropriate Liapunov's functional except in some special cases which belong to a class of linear homogeneous problems. Some authors exploit an energy-like functional in accord with the classical concept of stability due to the second or the direct method of Liapunov. In practice, the kinetic-like energy functionals as combinations of $L_{2}$ integrals of appropriate field variables are used to determine domains of stability. However, there are many situations where such an approach fails. The natural extension of the standard energy method is essentially the method of generalized energies used as Liapunov's functionals where by means of an integral inequality technique sharp quantitative stability bounds can be established. Stability or asymptotic stability is usually proved in terms of some positive definite norm by constructing Liapunov's functional whose positive definiteness with respect to the chosen norm together with negative definiteness of its time derivative along the trajectory of the system implies asymptotic stability with respect to the chosen norm. In the most general form such a procedure gives conditions for asymptotic stability in the large, since there is no restriction on the size of initial data, but only on their smoothness, no restrictions on the size of nonlinearity, but only some restriction on the form of nonlinearity. In general however, it is very difficult to consider the stability problem in the large, because the severe nonlinearity makes it very difficult to construct the appropriate Liapunov's functional. Problems of this kind can be resolved to some extent by considering the asymptotic stability in the first approximation as a result of consistent linearization of originally nonlinear problem. In this work we use an idea of constructing quadratic integral forms for which the Liapunov-like functionals are well suited for determining positive and negative definiteness of certain types of functionals. The method is quite general and can be applied to a large class of problems.

The paper is divided into two major parts. In the first part we discuss some general ideas within the established theoretical framework and define a general type of the problem to which the techniques described in the theoretical part of the paper can be applied. We discuss possibilities of constructing the Liapunov's functional in terms of a quadratic integral form and the application of the integral form in stability analysis based on the extended principles of the Liapunov's direct method. In the second part we treat a nonlinear damped wave equation and illustrate the application of theoretical apparatus developed in the first part.

\section{BASIC DEFINITIONS}

In what follows we give basic definitions needed for further development [28]. These definitions hold for a general set of PDEs of the form

$$
\partial_{t} u=g(x, u, \nabla u, \ldots) \quad: \Omega
$$

With the corresponding homogeneous boundary conditions 


$$
G(x, u, \ldots)=0 \quad: \partial \Omega
$$

However, in the course of the paper we apply the theory to a special set of semi-linear PDEs. In particular, we discuss semi-linear systems

$$
\partial_{t} u(x, t)=L_{x} u(x, t)+\mathcal{N}(u(x, t))
$$

Or semi-linear wave equations

$$
\partial_{t t} u(x, t)=L_{x} u(x, t)+\mathcal{N}(u(x, t))
$$

where $u \in \mathbb{R}^{N}$ depends on time $\mathbb{R} \in\left[t_{0}=, T \leq \infty\right]$. and $x \in \Omega \subset \mathbb{R}^{M}$ with the given sufficiently smooth initial data. The semi-linear operator is of the form

$$
\begin{aligned}
L_{x_{i}}(u)= & \sum_{j=1}^{N} a_{i j}(x) u_{j}+\sum_{j=1}^{N} \sum_{k=1}^{M} b_{i j}^{k}(x) \frac{\partial u_{j}}{\partial x_{k}}+ \\
& \sum_{j=1}^{N} \sum_{k=1}^{M} \sum_{l=1}^{M} c_{i j}^{k l}(x) \frac{\partial^{2} u_{j}}{\partial x_{k} \partial x_{l}}
\end{aligned}
$$

The corresponding initial and boundary conditions are given as

$$
\begin{aligned}
& u_{i 0}=u_{i}\left(x, t_{0}\right) \quad(i=1,2 \ldots N), \\
& \sum_{j=1}^{N}\left(A_{i j} u_{j}+\sum_{k=1}^{M} A_{i j}^{M} \frac{\partial u_{j}}{\partial x_{k}}\right)=0 \quad: \partial \Omega .
\end{aligned}
$$

$\mathcal{N}(u(x, t))$ is a nonlinear vector function well defined and continuous in its arguments in the domain of variation of all arguments contained in $\mathbb{R}^{N}$. In general, the solution of the nonlinear hyperbolic problem may not remain smooth for all time. We study the stability of admissible classical solutions, i.e., solutions which belong to the class of $C^{1}$ spatially differentiable functions. Coefficients $a_{i j}(x), b_{i j}^{k}(x), d_{i j}(x), e_{i j}^{k}(x)$ and $c_{i j}^{k l}(x)$ are continuous, partially differentiable functions of the class $C^{1}$ and $C^{2}$ respectively. We assume that for any initial data $u_{0}$ satisfying the required smoothness conditions there exists a unique solution of the boundary value problem $u\left(u_{0}, t_{0}, t\right)$ with the following properties: $u\left(u_{0}, t\right)$ is defined for all $t \geq t_{0} ; u\left(u_{0}, t\right)$ is continuous in $t$ and $u_{0} ; u\left(u_{0}, t_{0}\right)=u_{0}$ defines the initial state of the system. Then for fixed $u_{0}, u\left(u_{0}, t_{0}, t\right)$ defines a process, trajectory, motion or any other admissible state of the system described by a set of PDEs. If the problem is linear $(\mathcal{N}(u(x, t))=0)$ it is always possible to express the stability of a given solution $u(x, t)$ in terms of the stability of the null solution also called the zero solution, the trivial or the basic solution. Then the stability of every solution of the linear system can be determined by the stability of the null solution. If the system is nonlinear the behavior of the null solution cannot describe the behavior of all solutions. The classical stability definitions for the first approximation of consistently linearized systems [13], [21], [26] have to be extended and generalized as described for example in [2], [24], [28] and especially [27]. We investigate the stability of the zero solution of the problem that is formu-lated as follows. Let $\hat{u}(x, t)$ denote basic unperturbed motion (flow) and let $\tilde{u}(x, t)$ denote perturbed motion (flow). Then the difference

$$
u(x, t)=\tilde{u}(x, t)-\hat{u}(x, t)
$$

Defines the perturbation satisfying the homogeneous boundary conditions

$$
u(x, t)=0 \quad: \partial \Omega,
$$

Since in the process of perturbation we keep boundary conditions fixed. The leading equation for the evolution of disturbance is defined by a system of semi-linear PDEs.

\section{A. Function Spaces, Norms, and Metrics}

Let $L_{p}(\Omega), 1 \leq p \leq \infty$ designate the space of Lebesgue measurable functions $u$ defined on an open, bounded domain $\Omega \subset \mathbb{R}^{M}$ where $|u|^{p}$ are $L$-integrable over $\Omega$ and let $L_{2}(\Omega)$ be a space of square integrable functions over $\Omega$ with the corresponding norms

$$
L_{p}(\Omega):\|u\|_{L_{p}(\Omega)}=\left(\int_{\Omega}|u|^{p} d \Omega\right)^{1 / p}
$$

And

$$
L_{2}(\Omega):\|u\|_{L_{2}(\Omega)}^{2}=\int_{\Omega}|u|^{2} d \Omega
$$

Similarly, define the Sobolev space

$$
H_{1}(\Omega)=\left\{u \in L_{2}(\Omega): \nabla u \in L_{2}(\Omega)\right\}
$$

With elements that have one square integrable derivative over $\Omega$ with the corresponding $k=1$ norm

$$
\|u\|_{H_{1}(\Omega)}=\left(\|u\|_{L_{2}(\Omega)}^{2}+\sum_{i=1}^{M}\left\|\frac{\partial u}{\partial x_{i}}\right\|_{L_{2}(\Omega)}^{2}\right)^{1 / 2}
$$

According to the definition we will investigate the stability of the null solution which belongs to the subspace $H_{0}^{1}(\Omega)$ whose elements have one square integrable derivative and vanish on the boundary $\partial \Omega$ : 


$$
H_{0}^{1}(\Omega)=\left\{u \in H_{1}(\Omega): u=0 \text { on } \partial \Omega\right\} .
$$

\section{B. A Metric Space $\Phi$ with the Metric $\varrho$}

Since the stability definitions in their most general form refer to the abstract notion of a metric, neighborhood, and equivalence [21], [22], we first define the metric space $\Phi$ with the metric $\varrho$. Let

$$
\Phi=\left\{u\left(u_{0}, t_{0}, t\right) ; u_{0}=u\left(u_{0}, t_{0}\right) ; t \in\left[t_{0}, T \leq \infty\right]\right\}
$$

denote a function space where $u\left(u_{0}, t_{0}, t\right)$ describes a process, trajectory, motion or any other admissible state of the system described by a set of PDEs. The metric $\varrho$ is defined on $\Phi$ if for any two elements $\phi \in \Phi$ and $\psi \in \Phi$ there exists a real number $\varrho(\phi, \psi)$ with the following properties:

1) $\varrho(\phi, \psi)$ is a positive definite function $\varrho(\phi, \psi) \geq 0$; $\varrho(\phi, \psi)=0$ if $\phi=\psi$.

2) $\varrho(\phi, \psi)$ satisfies the triangle inequality $\varrho(\phi, \psi) \leq \varrho(\psi, \eta)+\varrho(\eta, \phi) \quad \forall \quad \eta \in \Phi$.

3) a symmetry condition: $\varrho(\phi, \psi)=\varrho(\psi, \phi)$.

Two metrics $\varrho_{1}$ and $\varrho_{2}$ are equivalent if there exist two positive real constants $a>0, b>0$ such that

$$
a \varrho_{1}(u, v)<\varrho_{2}(u, v)<b \varrho_{1}(u, v) \quad \forall(u, v) \in \Phi .
$$

Any such positive definite function can be used to define a neighborhood $S$ of a solution $u$ as an open ball with the center at $u$ and a radius $d$

$$
S(u, d)=\{u, v \in \Phi: \varrho(u, v)<d\} .
$$

In the same way we can define the neighborhood $S(0, d)$ of the trivial solution $u=0$.

\section{Choice of the Metric $\varrho$}

The relative freedom in choosing the positive definite function may have its drawback in the sense that for a given system one choice of the metric may imply stability, but not the other. The final choice of the metric depends on the particular problem. For example, if some local properties of the solution are investigated then a uniform metric such as $\sup _{\Omega}|u|$ should be employed. On the other hand, for the overall stability investigation over the domain $\Omega$ mean value integrals can be utilized so that the metric $\varrho$ belongs to the $L_{2}(\Omega)$ or $H_{1}(\Omega)$ spaces. These spaces make good choice since many useful relations which can be directly applied in stability analysis have been established [1], [20]. In particular, we will focus on the following two types of metrics:

$$
\begin{aligned}
\varrho & =\|u\|_{L_{2}(\Omega)}^{2}=\int_{\Omega} u^{T}(x, t) u(x, t) d x \\
& =\int_{\Omega} \sum_{i=1}^{N} u_{i}^{2} d x_{1} \ldots d x_{M}, \quad \varrho \in L_{2}(\Omega)
\end{aligned}
$$

And

$$
\begin{aligned}
\varrho & =\|u\|_{H_{1}(\Omega)}^{2}=\int_{\Omega}\left[u^{T}(x, t) u(x, t)+\nabla u(x, t)^{T} \nabla u(x, t)\right] d x \\
& =\int_{\Omega} \sum_{i=1}^{N}\left[u_{i}^{2}(x, t)+\sum_{j=1}^{M}\left(\frac{\partial u_{i}}{\partial x_{j}}\right)^{2}\right] d x_{1} \ldots d x_{M} .
\end{aligned}
$$

\section{FORMULATION OF THE LiaPUNOV'S DiRECT METHOD FOR STABILITY}

Stability analysis by the Liapunov's direct method has been originally developed for stability investigation of ordinary differential equations [3], [4], [13], [16], [21], [25], and later extended to partial differential equations [5], [15], [22], [26],

where authors dealt mostly with linear systems of PDEs and stability analysis in the first approximation, i.e., the stability analysis of the linearized systems. Nonlinear equations were treated in the works of [2], [11], [18], [23], [24], [28], and especially in the work of Sirazetdinov [27].

Before proceeding to the main theorems, we give the following stability definitions needed for further development. Let $\varepsilon$ and $\delta$ be real numbers. Referring to the metric space $\Phi$ we have the following general stability definitions:

Definition 3.1: The zero solution $u(x, t)=0$ of the initial boundary value problem is said to be stable with respect to $\varrho$ if for every $\varepsilon>0$ there exists a constant $\delta(\varepsilon)>0$ such that $\varrho\left(u_{0}, 0\right)<\delta \Leftrightarrow \varrho\left(u\left(u_{0}, t\right), 0\right)<\varepsilon \forall t \geq 0$.

Definition 3.2: The zero solution is said to be asymptotically stable with respect to the metric $\varrho$ if

1) the zero solution is stable.

2) all solutions which are bounded initially $\varrho\left(u_{0}, 0\right)<\delta$ remain bounded for all time so that $\varrho\left(u\left(u_{0}, t\right), 0\right)<\varepsilon \forall t \geq 0$ and $\lim _{t \rightarrow \infty} \varrho\left(u\left(u_{0}, t\right), 0\right)=0$.

Now we state a theorem on asymptotic stability which depends on the construction of the Liapunov's functional. A functional $V[u]$ defined on $\Phi$ which maps a vector function $u(x, t) \in \Phi$ into a scalar function of $t$ and which satisfies the conditions of the Liapunov's stability theorem is called the Liapunov's functional.

Theorem 3.3: Liapunov's Stability Theorem. (Liapunov's Direct Method). Suppose there exists a functional 
$\mathcal{V}[u(x, t)]=V(t)$ differentiable in $t$ along every solution curve $u(x, t)$ such that $V(0)=0$ and

1) $V$ is positive definite with respect to $\varrho$;

2) $V$ admits an infinitesimally small upper bound ;

3) $V\left(u\left(u_{0}, t\right)\right)$ is non-increasing for $t \geq 0 \forall u_{0} \in \mathcal{S}(0, d)$;

4) There exists a positive constant $d^{\prime}$ such that $0<d^{\prime} \leq d: V\left(u\left(u_{0}, t\right)\right) \rightarrow 0$ as $t \rightarrow \infty \forall u_{0} \in \mathcal{S}\left(0, d^{\prime}\right)$;

then the zero solution $u(x, t)$ is asymptotically stable with respect to the chosen metric $\varrho$. In practice we can prove (1-4) in three steps; in what follows $\alpha$ and $\gamma$ are positive constants:

1') $V(u)>\alpha \varrho^{2}(u, 0), \quad \alpha>0$;

$\left.2^{\prime}\right)|V(u)| \leq \gamma \varrho^{2}(u, 0), \quad \gamma>0$;

3') $d V(u) / d t \leq 0 \quad \forall \quad t \geq 0$;

where the time derivative along the trajectory is defined as

$$
\begin{gathered}
\frac{d V}{d t}=\lim _{\Delta t \rightarrow 0} \frac{1}{\Delta t}\left\{V\left[u\left(u_{0}, t_{0}, t+\Delta t\right), t+\Delta t\right]\right. \\
\left.-V\left[u\left(u_{0}, t_{0}, t\right), t\right]\right\} \leq 0 .
\end{gathered}
$$

Similar approach has been frequently used in many special cases. For example, for conservative systems we may choose $V=E=$ const as the total energy of the system and then from the principle of conservation of energy it follows that $d V\left(u\left(u_{0}, t\right)\right) / d t=0$. In linear systems the choice of $V$ similar to $\varrho$, i.e., $V \propto \varrho^{2}$ is usually utilized. For nonlinear systems $1^{\prime}, 2^{\prime}$ can be applied only in the close neighborhood $\mathcal{S}(0, d \ll 1)$ of the basic solution, whereas for nonconservative and highly nonlinear dissipative systems the proper choice of $V$ is much more difficult.

The Liapunov's direct method for stability analysis has the advantage that it does not require the explicit knowledge of the solution. However, there is no general way to find an appropriate Liapunov's Functional. Though choices of sign definite forms are many, the time derivative of LF along the trajectory of the system may exhibit such a complicated structure that the determination of the sign definiteness of the resulting form may become virtually impossible. We will discuss two particular choices of the metric which may serve as the Liapunov's functional. The first one is the classical square of the norm,

$$
V(x, t)=\int_{\Omega} u^{T}(x, t) u(x, t) d x=\varrho, \quad \varrho=\|u(x, t)\|_{L_{2}(\Omega)}^{2},
$$

whereas the other is just a generalization of the first

$$
V(x, t)=\int_{\Omega} u^{T}(x, t) F(x, t) u(x, t) d x, F(x, t)>0, \forall t \geq t_{0} .
$$

The kernel $F(x, t)$ represents a positive definite continuously differentiable operator on $x \in \Omega$ for every $t \geq t_{0}=0$.

For systems of nonlinear PDEs of a special type with $L_{x}$ denoting a linear operator and $\mathcal{N}$ representing the nonlinear vector function of its arguments such that $\mathcal{N}_{u=0}=0$

$$
\frac{\partial u(x, t)}{\partial t}=L_{x} u(x, t)+\mathcal{N}(t, x, u, \nabla u, \ldots),
$$

Sirazetdinov [27] established the following theorem:

Theorem 3.4 (Sirazetdinov). The zero solution $u \equiv 0$ of the nonlinear system is stable with respect to the metric $\varrho$ if the trivial solution of the corresponding linear system is asymptotically stable, $V(t)$ is continuous with respect to $\varrho$ with the time derivative which satisfies the condition

$$
\left(\frac{d V}{d t}\right)=\left(\frac{d V}{d t}\right)_{L}+\left(\frac{d V}{d t}\right)_{\mathcal{N}} \leq 0,
$$

where $(\cdot)_{L}$ and $(\cdot)_{\mathcal{N}}$ are time derivatives along the trajectory due to the linear and nonlinear part of the system, respectively. The nonlinear part of derivative is given as

$$
\begin{array}{r}
\left(\frac{d V}{d t}\right)_{\mathcal{N}}=\int_{\Omega_{x}} \int_{\Omega \xi}\left[u^{T}(x, t) F(x, \xi) \mathcal{N}\left(t, \xi, u, \nabla_{\xi}, \ldots\right)+\right. \\
\left.\left.\mathcal{N}^{T}\left(t, x, u, \nabla_{x}, \ldots\right) F(x, \xi) u(\xi, t)\right] d x d \xi\right] .
\end{array}
$$

The structure and derivation of the linear part of the time derivative for the particular choice of LF are both given in the next section. The nonlinear part of the derivative can be evaluated after the analysis for the linear part has been completed.

\section{CONSTRUCTION OF A Sign-DEFINITE INTEGRAL FORM As A LF}

To illustrate the procedure we first consider the linear part of the problem $(\mathcal{N}=0)$ for the operator $L_{x}$ and the quadratic integral form

$$
V=\int_{\Omega_{x}} \int_{\Omega_{\xi}} \sum_{i, j=1}^{N} u_{i}(x, t) f_{i j}(x, \xi) u_{j}(\xi, t) d x d \xi,
$$

where the operator $f_{i j}(x, \xi) \in C^{2}$ is twice continuously differrentiable with respect to $x, \xi \in \mathbb{R}^{M}$. Calculation of the time derivative along the trajectory of the system $\partial_{t} u=L_{x} u$ yields 


$$
\begin{array}{r}
\frac{d V}{d t}=\int_{\Omega_{x}} \int_{\Omega_{\xi}} \sum_{i, j=1}^{N}\left(\frac{\partial u_{i}(x, t)}{\partial t} f_{i j}(x, \xi) u_{j}(\xi, t)\right. \\
\left.+u_{i}(x, t) f_{i j}(x, \xi) \frac{\partial u_{j}(\xi, t)}{\partial t}\right) d x d \xi .
\end{array}
$$

After integrating by parts and using Gauss divergence theorem in $\mathbb{R}^{M}$ we obtain the following form of the time derivative of the $\mathrm{LF}$

$$
\frac{d V}{d t}=\int_{\Omega_{x}} \int_{\Omega_{\xi}} \sum_{i, j=1}^{N} u_{i}(x, t) L_{i j}^{*}(f) u_{j}(\xi, t) d x d \xi .
$$

In previous expression the adjoint operator $L_{i j}^{*}$ is defined as

$$
L_{i j}^{*}(f)=\ell_{i j}(f)+\ell_{j i}(f)
$$

where the corresponding operators $\ell_{i j}(f)$ and $\ell_{j i}(f)$ take the following forms

$$
\begin{aligned}
\ell_{i j}(f) & =\sum_{k=1}^{N} f_{i k} a_{k j}(\xi)-\sum_{k=1}^{N} \sum_{l=1}^{M} \frac{\partial f_{i k} b_{k j}^{l}(\xi)}{\partial \xi_{l}} \\
& +\sum_{k=1}^{N} \sum_{l=1}^{M} \sum_{p=1}^{M} \frac{\partial^{2} f_{i k} c_{k j}^{l p}(\xi)}{\partial \xi_{l} \partial \xi_{p}}
\end{aligned}
$$

And

$$
\begin{aligned}
\ell_{j i}(f) & =\sum_{k=1}^{N} f_{k j} a_{k i}(x)-\sum_{k=1}^{N} \sum_{l=1}^{M} \frac{\partial f_{k j} b_{k i}^{l}(x)}{\partial x_{l}} \\
& +\sum_{k=1}^{N} \sum_{l=1}^{M} \sum_{p=1}^{M} \frac{\partial^{2} c_{k j}^{l p}(x) f_{k i}}{\partial x_{l} \partial x_{p}} .
\end{aligned}
$$

Here we have considered that $u \in H_{0}^{1}(\Omega)$, so the boundary conditions for functions $f_{i j}$ can be chosen in such a way to make surface integrals on $\partial \Omega$ vanish.

Now explore a functional $\mathcal{U}[u(x, t)]$ that satisfies

$$
\frac{d V}{d t}=\mathcal{U}
$$

By taking into account the chosen quadratic integral form $V$ as the LF and by considering the structure of calculated time derivative $d V / d t$

$$
\frac{d V}{d t}=\int_{\Omega_{x}} \int_{\Omega_{\xi}} \sum_{i, j=1}^{N} u_{i}(x, t) L_{i j}^{*}(f) u_{j}(\xi, t) d x d \xi,
$$

We can make a suitable choice for $\mathcal{U}$ in the following form

$$
\mathcal{U}=\int_{\Omega_{x}} \int_{\Omega_{\xi}} \sum_{i, j=1}^{N} u_{i}(x, t) \mathcal{U}_{i j}(x, \xi) u_{j}(\xi, t) d x d \xi
$$

Comparing expressions for $d V / d t$ and $\mathcal{U}$ yields the boundary

value problem for the unknown set $f$ in terms of a given right hand side $\mathcal{U}$ :

$$
L_{i j}^{*}(f)=\mathcal{U}_{i j}
$$

To construct a quadratic integral form as a LF actually means to solve for the unknown functions $f_{i j}$ for the given right hand side $\mathcal{U}_{i j}$. Once the components of $F(x, \xi)$ are known, we first have to prove under what conditions $V$ is positive definite. By choosing components of the quadratic form $\mathcal{U}$ so that $\mathcal{U}$ is negative definite, we can apply the Theorem 3.4 and proceed with proving $\dot{V}=\dot{V}_{L}+\dot{V}_{\mathcal{N}} \leq 0$ to establish the stability conditions including the influence of nonlinear terms.

\section{A Nonlinear WAVE EQUATION In A Dissipative MEDIUM}

Consider a nonlinear wave equation [26] of a uniform string with the normalized length $\pi$ with fixed ends $u(0, t)=0$ and $u(\pi, t)=0$, where $u(x, t)$ is the transversal displacement from equilibrium position $u=0$ :

$$
\frac{\partial^{2} u}{\partial t^{2}}=a \frac{\partial^{2} u}{\partial x^{2}}+b \frac{\partial u}{\partial t}+\psi_{1}\left(t, x, u, u_{t}\right)
$$

Here $a$ and $b$ are real constants, $\psi_{1}$ is a smooth nonlinear function with bounded variation defined on the domain $0 \leq t, 0 \leq x \leq \pi,-\infty<u<\infty$. Rewrite the equation as a first order system

$$
\begin{aligned}
\frac{\partial u_{1}}{\partial t} & =a \frac{\partial^{2} u_{2}}{\partial x^{2}}+b u_{1}+\psi_{1}\left(t, x, u_{2}, u_{1}\right) \\
\frac{\partial u_{2}}{\partial t} & =u_{1}
\end{aligned}
$$

To put the system in operator form $\partial_{t} u=L_{X} u+\mathcal{N}$ denote

$$
u=\left(\begin{array}{l}
u_{1}(x, t) \\
u_{2}(x, t)
\end{array}\right), \quad L_{X}=\left[\begin{array}{cc}
b & a \frac{\partial^{2}}{\partial x^{2}} \\
1 & 0
\end{array}\right], \quad \mathcal{N}=\left(\begin{array}{c}
\psi_{1}\left(t, x, u_{1}, u_{2}\right) \\
0
\end{array}\right)
$$

We investigate the stability with respect to the metric $\varrho$ 


$$
\varrho=\int_{0}^{\pi}\left[\left(\frac{\partial u}{\partial t}\right)^{2}+\left(\frac{\partial u}{\partial x}\right)^{2}\right] d x=\int_{0}^{\pi}\left[u_{1}^{2}+\left(\frac{\partial u_{2}}{\partial x}\right)^{2}\right] d x
$$

And define the Liapunov's function $V$ as

$$
\begin{aligned}
& V=\int_{0}^{\pi} \int_{0}^{\pi}\left[u_{1}(x, t) f_{11}(x, \xi) u_{1}(\xi, t)+u_{1}(x, t) f_{12}(x, \xi) \frac{\partial u_{2}(\xi, t)}{\partial \xi}\right. \\
& \left.+\frac{\partial u_{2}(x, t)}{\partial x} f_{21}(x, \xi) u_{1}(\xi, t)+\frac{\partial u_{2}(x, t)}{\partial x} f_{22}(x, \xi) \frac{\partial u_{2}(\xi, t)}{\partial \xi}\right] d x d \xi
\end{aligned}
$$

In connection with the corresponding integral form $\mathcal{U}$

$$
\begin{aligned}
\mathcal{U} & =\int_{0}^{\pi} \int_{0}^{\pi}\left[u_{1}(x, t) \mathcal{U}_{11}(x, \xi) u_{1}(\xi, t)\right. \\
& \left.+\frac{\partial u_{2}(x, t)}{\partial x} \mathcal{U}_{22}(x, \xi) \frac{\partial u_{2}(\xi, t)}{\partial \xi}\right] d x d \xi
\end{aligned}
$$

where the coefficients of the integral form $\mathcal{U}$ are expressed in terms of series

$$
\mathcal{U}_{11}=\sum_{s=1}^{\infty} \sum_{r=1}^{\infty} \breve{\mathcal{U}}_{s r} \sin s x \sin r \xi, \quad \mathcal{U}_{22}=\sum_{s=1}^{\infty} \sum_{r=1}^{\infty} \widehat{\mathcal{U}}_{s r} \cos s x \cos r \xi .
$$

We seek the solution of the adjoint linear system of PDEs of the form $L_{i j}^{*}(f)=\mathcal{U}_{i j}$ in terms of series. By extracting the diagonally dominant modes with transformations $\breve{\mathcal{U}}_{r s}=\breve{\mathcal{U}}_{s} \delta_{r s}$ and $\hat{\mathcal{U}}_{r s}=\hat{\mathcal{U}}_{s} \delta_{r s}$, where $\delta_{r s}$ is the Kronecker-delta with the properties $\left(\delta_{r s}=1\right.$ for $r=s$ and $\delta_{r s}=0$ for $\left.r \neq s\right)$, we are able to construct the operator $F(x, \xi)$ as

$$
F(x, \xi)=\left[\begin{array}{ll}
f_{11}(x, \xi) & f_{12}(x, \xi) \\
f_{21}(x, \xi) & f_{22}(x, \xi)
\end{array}\right] .
$$

Here the elements of operator $F(x, \xi)$ are given as

$$
\begin{aligned}
& f_{11}(x, \xi)=\frac{1}{2 b} \sum_{s=1}^{\infty}\left(\breve{\mathcal{U}}_{s}+\widehat{\mathcal{U}}_{s}\right) \sin s x \sin s \xi, \\
& f_{12}(x, \xi)=\frac{1}{2 b} \sum_{s=1}^{\infty}\left(-\frac{\widehat{\mathcal{U}}_{s}}{s}\right) \sin s x \cos s \xi, \\
& f_{21}(x, \xi)=\frac{1}{2 b} \sum_{s=1}^{\infty}\left(-\frac{\overline{\mathcal{U}}_{s}}{s}\right) \cos s x \sin s \xi, \\
& f_{22}(x, \xi)=\frac{1}{2 b} \sum_{s=1}^{\infty}\left(\breve{\mathcal{U}}_{s}+\left(1+\frac{b^{2}}{s^{2}}\right) \overline{\mathcal{U}}_{s}\right) \cos s x \cos s \xi
\end{aligned}
$$

\section{A. The Linear Part}

A formal solution of the linear part of the equation in terms of series can be written as

$$
u_{1}=\sum_{s=1}^{\infty} \alpha_{s}(t) \sin s x, \quad u_{2}=\sum_{s=1}^{\infty} \beta_{s}(t) \sin s x,
$$

where $\left\{\alpha_{s}\right\}$ and $\left\{\beta_{s}\right\}$ are two time dependent arrays of infinite order. Substituting the solution for the linear part of the problem in expressions for the metric $\varrho$, after performing required domain integrals, yields

$$
\varrho_{L}=\frac{\pi}{2} \sum_{s=1}^{\infty}\left(\alpha_{s}^{2}+s^{2} \beta_{s}^{2}\right)
$$

Similarly, having employed some fundamental exercises among infinite sequences and series [17], the LF for the linear part of the problem takes on the following form

$$
\begin{aligned}
V & =\frac{\pi^{2}}{8 b} \sum_{s=1}^{\infty}\left[\left(\alpha_{s}^{2}+s^{2} \beta_{s}^{2}\right) \breve{\mathcal{U}}_{s}\right. \\
& \left.+\left(\alpha_{s}^{2}-2 \alpha_{s} \beta_{s}+\left(b^{2}+s^{2}\right) \beta_{s}^{2}\right) \widehat{\mathcal{U}}_{s}\right] .
\end{aligned}
$$

By using certain algebraic inequalities [17],[18] wellsuited for the problem at hand, one can prove that if $\breve{\mathcal{U}}_{s}$ and $\overline{\mathcal{U}}_{s}$ are bounded then $V$ is also bounded with respect to the metric $\varrho_{L}, \quad$ i.e., $\quad|V| \leq \mathrm{c}_{1} \varrho_{L}$. Moreover, for $\left(\breve{\mathcal{U}}_{s} / b>0, \widehat{\mathcal{U}}_{s} / b>0\right)$ it can be shown that the LF is positive definite with respect to the metric $\varrho_{L}: V>\mathrm{c}_{2} \varrho_{L}>0$, where $\mathrm{c}_{1}$ and $\mathrm{c}_{2}$ are positive constants.

In order to construct the appropriate structure of integral form $\mathcal{U}$, as a right hand side of equation representing the time derivative of LF, we employ the transformation $\breve{\mathcal{U}}_{r s}=(-2 / \pi) \delta_{r s}$ and $\hat{\mathcal{U}}_{r s}=(2 / \pi) \delta_{r s}$ in expressions for $\mathcal{U}_{11}$ and $\mathcal{U}_{22}$ and consider the series representation [6], [7], [10]

$$
\begin{aligned}
& \mathcal{U}_{11}=-\frac{2}{\pi} \lim _{N \rightarrow \infty} \sum_{s=1}^{N} \sin s x \sin s \xi=-\delta(x-\xi), \\
& \mathcal{U}_{22}=\frac{2}{\pi} \lim _{N \rightarrow \infty} \sum_{s=1}^{N} \cos s x \cos s \xi=-\delta(x-\xi),
\end{aligned}
$$

where $\delta(x-\xi)$ is the Dirac-delta function. Using the wellknown properties of the Dirac-delta function [8], [12], [14] in expression which defines the structure of the integral form $\mathcal{U}$ yields 


$$
\mathcal{U}=-\int_{0}^{\pi}\left[u_{1}^{2}+\left(\frac{\partial u_{2}}{\partial x}\right)^{2}\right] d x=-\varrho_{L}<0
$$

From where it follows that the time derivative along the trajectory of the linear part of the system is negative definite, i.e., $(d V / d t)_{L}=\mathcal{U}<0$, hence the linear part of the system is asymptotically stable.

\section{B. The Nonlinear Part}

The nonlinear part of the time derivative along the trajectory of perturbed solution is defined as

$$
\left(\frac{d V}{d t}\right)_{\mathcal{N}}=\int_{\Omega_{x}} \int_{\Omega_{\xi}}[\mathcal{N} F u+u F \mathcal{N}] d x d \xi
$$

From an explicit form of previous relation we can establish the estimate [27]

$$
\begin{aligned}
& \left(\frac{d V}{d t}\right)_{\mathcal{N}}=\int_{0}^{\pi} \int_{0}^{\pi}\left[u_{1}(x, t) f_{11}(x, \xi) \psi_{1}(\xi, t, \ldots)\right. \\
& +\psi_{1}(x, t, \ldots) f_{11}(x, \xi) u_{1}(\xi, t)+\psi_{1}(x, t, \ldots) f_{12}(x, \xi) \frac{\partial u_{2}(\xi, t)}{\partial \xi} \\
& \left.+\frac{\partial u_{2}(x, t)}{\partial x} f_{21}(x, \xi) \psi_{1}(\xi, t, \ldots)\right] d x d \xi \leqslant \max \left[\left|f_{11}\right|,\left|f_{12}\right|,\left|f_{21}\right|\right] \times \\
& \times\left[\int_{0}^{\pi}\left|u_{1}\right| d x+\int_{0}^{\pi}\left|\frac{\partial u_{2}}{\partial x}\right| d x\right] \int_{0}^{\pi}\left|\psi_{1}\right| d x .
\end{aligned}
$$

If the nonlinearity of $\psi_{1}$ is such that we can prove the following inequality expressed in terms of absolute values

$$
\frac{\left|(d V / d t)_{\mathcal{N}}\right|}{|\mathcal{U}|}<1
$$

Then the basic solution is stable with respect to the metric $\varrho_{L}$ where the effect of nonlinearity has been included in the estimate. To obtain the qualitative bound for the magnitude of nonlinearity the particular form of $\psi_{1}$ has to be specified. Subsequently, by using an integral inequality technique, the sharper bound for the threshold of stability can be established.

\section{REFERENCES}

[1] R. A. Adams, Sobolev Spaces. Academic Press, 1975.

[2] T. K. Caughey and J. Ellison, "Existence, uniqueness and stability of solutions of a class of nonlinear partial differential Equations," J. Math. Anal. Appl., vol. 51, pp. 1-32, 1975.

[3] N. G. Chetayev, The Stability of Motion. Pergamon Press, 1961.

[4] M. Cotsaftis, "On Stability of Motion," J. Inst. Math. Appl., vol. 5, pp. $19-54,1968$.

[5] C. M. Dafermos and W. J. Hrusa, "Energy methods for quasilinear hyperbolic initial-boundary value problems. Applications to elastodynamics," Arch. Ration. Mech. An., vol. 87, pp. 267-292, 1985.
[6] J. J. Duistermaat and J. A. C. Kolk, Distributions. Theory and Applications. Springer, 2010.

[7] R. Estrada and R. P. Kanwal, A Distributional Approach to Asymptotics. Theory and Applications. $2^{\text {nd }}$ ed., Birkhauser, 2002.

[8] R. P. Kanwal, Generalized Functions. Theory and Technique. $2^{\text {nd }}$ ed., Birkhauser, 1998.

[9] M. M. Lavrentjev, V. G. Romanov and S. P. Shishatskii, Ill-posed Problems of Mathematical Physics and Analysis. Translations of Mathematical Monographs, vol. 64, American Mathematical Society, 1980

[10] M. J. Lighthill, An Introduction to Fourier Analysis and Generalized Functions. Cambridge Univ. Press, 1989.

[11] W. Eckhaus, Studies in Non-linear Stability Theory. Springer Tracts in Natural Philosophy, vol. 6, Springer, 1965.

[12] G. Friedlander and M. Joshi, Introduction to The Theory of Distributions $2^{\text {nd }}$ ed., Cambridge Univ. Press, 1998.

[13] W. Hahn, Stability of Motion. Springer, 1967.

[14] D. S. Jones, The Theory of Generalized Functions. Cambridge Univ. Press, 1982.

[15] R. J. Knops and E. W. Wilkes, "On Movchan's theorems for stability of continuous Systems," Int. J. Eng. Sci., vol. 4, pp. 303-329, 1966.

[16] N. N. Krasovskii, Stability of Motion. Applications of Lyapunov's second method to differential systems and equations with delay. Stanford Univ. Press, 1963.

[17] K. Knopp, Infinite Sequences and Series. Dover Publications, Inc., 1956.

[18] V. Lakshmikantham and S. Leela, Differential and Integral Inequalities. Vol. 1, Vol. 2, Academic Press, 1969.

[19] V. Lakshmikantham, S. Leela, and A. A. Martynyuk, Practical Stability of Nonlinear Systems. World Scientific, 1990.

[20] V. Lakshmikantham and X. Z. Liu, Stability Analysis in Terms of Two Measures. World Scientific, 1993.

[21] J. LaSalle and S. Lefschetz, Stability by Liapunov's Direct Method. Academic Press, 1961.

[22] A. A. Movchan, "Stability of processes with respect to two metrics," Appl. Math. Mech., vol. 24, pp. 1506-1524, 1960.

[23] J. M. Skowronski, Nonlinear Liapunov Dynamics. World Scientific, 1990.

[24] A. J. Pritchard, “On nonlinear stability theory,” Q. Appl. Math., vol. 27, pp. 531-536, 1970.

[25] N. Rouche, P. Habets and M. Laloy, Stability Theory by Liapunov's Direct Method. Appl. Math. Sci., vol. 22, Springer, 1977.

[26] R. T. Shield, "On the stability of linear continuous systems," Z. Angew. Math. Phys., vol. 16, pp. 649-686, 1965.

[27] T. K. Sirazetdinov and P. K. Semenov, "Stability analysis of systems with continuous parameters by the method of Liapunov's Functions," in Stability of Motion, V. M. Matrosov and V. D. Irtegov, Eds. Nauka, Novosibirsk, 1985, pp. 3-22, in Russian.

[28] V. I. Zubov, Methods of A. M. Liapunov and their Application. P. Noordhoff, 1964. 